\title{
Precipitation of Gas Condensates Under the Influence of Capillary Pressure in Porous Media
}

\author{
POTSCH, Klaus T., OMV
}

Paper presented at the 5th European Conference on the Mathematics of Oil Recovery, Leoben, Austria, 3-6 Sept. 1996

\begin{abstract}
:
This paper describes a method of calculating the influence of the capillary pressure on the phase distribution in a porous medium. The theory predicts an increase of liquid drop out. Because of the interface between vapor and liquid the pressures in both phases are not equal. To calculate the thermal equilibrium in the presence of connate water one has to know which of the liquid phases is the wetting one. Both the water wet and the oil wet cases are considered. For pressures sufficiently differing from the dew point pressure an asymptotic theory (an expansion of a small parameter which is the ratio between the capillary pressure and the actual pressure) can be applied. In the vicinity of the dew point, this approach can break down under certain circumstances when the capillary forces grow out of bounds. In this case, the phase calculations have to be performed by means of an iteration technique. The results are applied to an Austrian gas condensate reservoir.
\end{abstract}

\section{INTRODUCTION:}

Gas and oil reservoirs differ from each other inasmuch as the production from low permeability reservoir rocks is still economic in gas reservoirs. Gas condensates with their retrograde phase behavior lie between these cases. First, samples from a gas condensate originate usually from the mobile gas phase. It is often reported that the gas condensate reservoir has an underlying oil rim as is the case in an Austrian gas condensate reservoir. In order to estimate the reserves of hydrocarbons properly, one has to know, at the given pressure and composition of the gas phase, the quantity and the quality of the liquid that is in thermodynamic equilibrium with the gas phase. It should not be assumed that an overall composition can be obtained from sampling.

\section{PHASE EQUILIBRIUM CALCULATIONS:}

The theory of phase equilibrium calculations is well established in the literature [1],[2]. The general set of equations for a two phase system (gas and liquid phase) consists of:

mechanical equilibrium

$p^{g a s}=p^{\text {liq }}$

thermal equilibrium

$T^{g a s}=T^{l i q}$

chemical equilibrium

$\mu_{k}^{g a s}\left(p^{g a s}, T^{g a s}, c_{k}^{g a s}\right)=\mu_{k}^{l i q}\left(p^{l i q}, T^{l i q}, c_{k}^{l i q}\right)$

with $p$ denoting the pressure, $T$ the absolute temperature and $c$ the molar concentration of the system made up of $K$ components. The superscripts symbolize the phase. Instead of the chemical potential $\mu_{k}$ the fugacities $f_{k}$ can be used. The system of equations contains as well the mass conservation of each component

$c_{k}^{\text {tot }}=(1-\alpha) c_{k}^{\text {gas }}+\alpha c_{k}^{\text {liq }}$

with $\alpha$ being the molar fraction of the liquid phase, which is determined by the Rachford-Rice equation [3]

$\sum_{k} \frac{c_{k}^{t o t}\left(q_{k}-1\right)}{1+\alpha\left(q_{k}-1\right)}=0$

The $q_{k}$ are the equilibrium ratios (usually known as the K-values) and are defined here differently as 
$q_{k}=\frac{c_{k}^{l i q}}{c_{k}^{g a s}}$

Finally, the norm conditions for the concentrations of the components complete the system

$\sum_{k} c_{k}^{g a s}=\sum_{k} c_{k}^{\text {liq }}=1$

In textbooks, there is no mention made of the shape of the interface between the two phases. The assumption is tacitly made that the interface has no curvature. In a porous medium, one of the phases is the more, or less, wetting phase. This fact introduces a nonzero curvature and, hence, also the influence of the capillary pressure which has to be accounted for. Equ. (1) has to be modified to

$p^{g a s}=p^{l i q}+p_{c}$

The capillary pressure is determined by the geometry of the porous medium. Petrophysical investigations yield the dimensionless Leverett $\mathrm{J}$ function from which the capillary pressure is best calculated [4]

$p^{c a p}=p_{r e f}^{c a p} J(S), \quad p_{r e f}^{c a p}=\sigma \cos \vartheta \sqrt{\phi / k}$

$\sigma$ is the interfacial tension which depends on the composition of the phases, $\vartheta$ the contact angle and $S_{w}$ is the saturation of the wetting phase (liquid) which relates to $\alpha$ via the relationship

$S_{w}=\frac{1}{1+C(1 / \alpha-1)}, \quad C=\frac{\rho^{\text {liq }} M^{g a s}}{\rho^{g a s} M^{l i q}} \ldots \ldots$

The total number of linear independent equations is $3 K+5$. They contain $4 K+6$ variables: $p^{g a s}$, $p^{\text {jiq }}$, $p^{\text {cap }}, T^{\text {gas }}, T^{\text {liq }}, \alpha, c_{k}{ }^{\text {tot }}, c_{k}{ }^{\text {gas }}, c_{k}{ }_{k}^{\text {liq }}, q_{k}$. That means $K+1$ variables have to be prescribed in order to render the system uniquely solvable. In practice, one would have data like pgas, $T^{g a s}, c_{k}{ }^{\text {tot }}$ in the laboratory case and pgas, Tgas, $c_{k}$ gas in the field case. In this work, the unknowns are chosen to be the $q_{k}$. The mass balance of the components allows for an easy calculation of the phase concentrations.

\section{CAPILLARY PRESSURE:}

Gas condensate systems necessitate the consideration of the capillary pressure. The pore space in which the condensate is encountered underground will have the capillary forces act on the interface of the phases. This will be the case sooner or later in the life of the reservoir, especially at low permeabilities (small pore throat radii). On the other hand, the investigation of the PVT behavior is carried out without the influence of capillary forces. The reservoir engineer may wonder if the laboratory results are representative of the processes going on in the reservoir. The main goal of this investigation is to work out the differences caused by the capillary forces when the number of moles and the volume that the gas condensate occupies are kept constant.

We consider here the easier case that the gas condensate forms only two phases, namely gas and liquid. Automatically the capillary pressure comes into play if the interface between the phases is curved. Consequently, the pressures in the two phases are not equal anymore (8) and the equilibrium condition (3) - now formulated using the fugacities - reads

$$
\begin{aligned}
& f_{k}^{g a s}\left(p^{g a s}, T^{g a s}, c_{k}^{g a s}\right)= \\
& \quad f_{k}^{l i q}\left(p^{g a s}-p^{c a p}, T^{l i q}, c_{k}^{l i q}\right)
\end{aligned}
$$

The capillary pressure introduces another difficulty into the phase calculations which are already nonlinear. In the case without capillary pressure, for a given pressure, temperature and composition of the overall system, equ. (11) determines the equilibrium ratios $q_{k}$ and the Rachford-Rice equ. (5) finally yields the mole fraction of the two phases, or the saturation of one phase. In order to start this iteration loop, in the case of the capillary pressure, one has to know the latter in advance. Since the capillary pressure itself depends on the fraction of the phases through equ. (9), the output of the phase calculation would be needed as an entry quantity.

\section{Several possibilities have to be considered:}

\section{Presence of an initial water saturation}

1.1 Water wet case. Fig.1 illustrates the occupation of the small pores by water. The maximum possible capillary pressure is given by $p^{c a p}\left(S_{w i}\right)$. This value will be true for infinitesimally small liquid drop out.

1.2 Intermediate wet case. After passing through the dew point line into the two phase region the liquid condensate phase is in competition with the water. As the water first occupies the pore walls and small pores, relatively low capillary pressures will be encountered.

1.3 Liquid condensate wet case. Higher values of capillary pressure are expected when the liquid 
hydrocarbons, after condensation, make the water recede from part of its original positions.

2. Absence of an initial water saturation. This is the worst case. Even the smallest pores are open for receiving the liquid drop out. Any value of capillary pressure, especially very high ones for small liquid fractions, are possible.

The actual capillary pressure is dependent on the amount of liquid drop out. A first estimate can be obtained by calculating the liquid drop out without porous medium in areas not too close to the dew point.

Around the dew point the capillary pressure grow's out of bounds. There exists a limit to which the capillary pressure can grow. It seems physically plausible that the pressure of the liquid phase cannot drop below zero although in the literature some authors have a different opinion [5]. That makes the capillary pressure equal to the pressure of the gas phase. With this assumption one can calculate a minimum liquid saturation that is of course pressure dependent.

\section{LINEAR CASE:}

In most of the cases, an approximation alleviates the complicated nonlinear situation. If the capillary forces are small in comparison to the actual pressure of the gas phase, then one can try an expansion of equ. (11) in powers of the ratio of the capillary pressure to the pressure of the gas phase. This renders the nonlinear system into a linear one. This approach has been taken in [6],[7]. This work will look at the linearization in more detail and at the assumptions and conditions made in general.

Let the capillary pressure be given as in equ.(9). The necessary small parameter is the ratio between the reference capillary pressure and the pressure the system would have without being contained inside a porous medium

$\varepsilon=\frac{p_{r e f}^{c a p}}{p_{0}}$

Let us look at the order of magnitude which $\varepsilon$ is usually expected to have. Fig. 2 shows graphically the dependance of $\varepsilon$ from IFT, permeability and reservoir pressure. These values of $\varepsilon$ hold under the assumption that the J-function is also of the order $O(1)$. For the above mentioned cases 1.3 and 2 . this might not be valid anymore.

Some general assumptions have to be explained before describing the calculation procedure. Firstly, the temperature is considered constant in both phases. Secondly, the balance equations are formulated for one mole. Thirdly, when the comparison is made between the case where the fluid is contained in a cell (nonporous case) and the case where the fluid resides in a porous medium (reservoir, porous case), the volume is considered the same.

The suggested expansion in terms of the small parameter will be introduced to all the variables involved in the phase equilibrium calculation. Only terms linear in $\varepsilon$ will be retained

$$
\begin{aligned}
& c_{k}^{g a s}=c_{0 k}^{g a s}+\varepsilon c_{1 k}^{g a s} \\
& c_{k}^{l i q}=c_{0 k}^{l i q}+\varepsilon c_{1 k}^{l i q} \\
& q_{k}=q_{k 0}+\varepsilon q_{1 k} \\
& \alpha=\alpha_{0}+\varepsilon \alpha_{1}
\end{aligned}
$$

A consequence of the third assumption is that if additional precipitation takes place in the porous medium, the pressure of the gas phase is expected to be lower. The same correction term has to be included in the liquid phase

$$
\begin{aligned}
& p^{g a s}=p_{0}(1-\varepsilon \Delta p) \\
& p^{\text {liq }}=p_{0}(1-\varepsilon \Delta p-\varepsilon J)
\end{aligned}
$$

Starting point for the calculation of the phase equilibrium is equ. (11). After inserting equs. (13) and (14) we get

$f_{k}^{g a s}\left(p_{0}(1-\varepsilon \Delta p), c_{0 j}^{g a s}+\varepsilon c_{1 j}^{g a s}\right)=$

$f_{k}^{l i q}\left(p_{0}(1-\varepsilon \Delta p-\varepsilon J), c_{0 j}^{l i q}+\varepsilon c_{1 j}^{l i q}\right)$

$k, j=1, \ldots, K$.

It turns out to be convenient to use the $q_{1 k}$ as the independent variables. After some arithmetic we arrive at

$$
p_{0} J\left(\alpha_{0}\right) \frac{\partial f_{k}^{l i q}}{\partial p}=\sum_{j}\left(\frac{\partial f_{k}^{l i q}}{\partial q_{j}}-\frac{\partial f_{k}^{g a s}}{\partial q_{j}}\right) q_{j} .
$$

The mass balance supplies the expressions for the concentrations 
$D_{k}=1+\alpha_{0}\left(q_{0 k}-1\right), \quad q_{0 k}=\frac{c_{0 k}^{l i q}}{c_{0 k}^{g a s}}$

$\frac{c_{1 k}^{g a s}}{c_{0 k}^{g a s}}=-\frac{\alpha_{0} q_{1 k}+\alpha_{1}\left(q_{0 k}-1\right)}{D_{k}}$,

$\frac{c_{1 k}^{l i q}}{c_{0 k}^{l i q}}=\frac{\left(1-\alpha_{0}\right) q_{1 k}-\alpha_{1} q_{0 k}\left(q_{0 k}-1\right)}{D_{k} q_{0 k}}$

The small increase in liquid drop out is calculated from the Rachford-Rice equation (5)

$$
\alpha_{1}=\frac{\sum_{k} \frac{c_{k}^{t o t}\left(q_{0 k}-1\right)}{D_{k}} \frac{q_{1 k}}{q_{0 k}}\left(1-\alpha_{0} \frac{q_{0 k}}{D_{k}}\right)}{\sum_{k} \frac{c_{k}^{\text {tot }}\left(q_{0 k}-1\right)^{2}}{D_{k}{ }^{2}}} \ldots
$$

The above mentioned third assumption of constant volume supplies the final expression for $\Delta p$ assuming that the compressibility factors of gas and liquid are only affected by the capillary effect in a negligible way

$\Delta p=\frac{\alpha_{1}\left(Z^{g a s}-Z^{l i q}\right)}{\left(1-\alpha_{0}\right) Z^{g a s}+\alpha_{0} Z^{i q q}}$

\section{NONLINEAR CASE:}

The nonlinear case consists of the equ. (1)-(10). The relevant quantities are sought in an iterative way. There is an important difference between the 'porous' and the 'nonporous' case. For both cases the $K$ equilibrium ratios $q_{k}$ are taken as independent variables. The iteration procedure linearizes the equation for the chemical equilibrium (expansion into a Taylor series and truncation after the linear tems)

$$
\begin{aligned}
& q_{j}^{(n+1)}=q_{j}^{(n)}+\sum_{k}\left(\mu_{k}^{g a s}-\mu_{k}^{g a s}\right) \\
& \left(\frac{\partial\left(\mu_{k}^{g a s}-\mu_{k}^{g a s}\right)}{\partial q_{j}}\right)^{-1}
\end{aligned}
$$

The superscript $n$ denotes the number of iterations. As mentioned above the chemical potentials depend on the concentrations and the molar fraction $a$ of the liquid phase via the Rachford-Rice equ.(5). The 'nonporous' case is a problem of $K$ and 1 variables: the $q_{k}$ and $a$ are evaluated consecutively and update the concentrations and the chemical potentials.
The 'porous' case posesses, in principle, $K+1$ independent variables because the pressure of the liquid phase depends on the liquid molar fraction. A similar approach leads to the desired iteration routine; at every step of calculating a new set of equilibrium ratios $q_{k}$ in equ.(20) one has to update the molar fraction $a$ and the pressure of the liquid phase. At very low values of $a$ (no wetting water phase present or liquid condensate phase is wetting) one has to make sure that the estimate of the liquid phase pressure does not reach physically impossible negative values.

\section{NUMERICAL CALCULATIONS:}

To study the effect of capillary pressure on the thermodynamic equilibrium a simple system consisting of two components and the overall compositions of $C_{1}\left(c^{t o t}=0.8\right)$ and $n C_{5}\left(c^{\text {tot }}=0.2\right)$ was chosen. The modelling of the thermodynamic equilibrium was done with a cubic equation of state (Peng-Robinson).

The 'model' porous medium has the following properties:

$\begin{array}{ll}\text { porosity } & 0.2 \\ \text { permeability } & 0.001 \mu \mathrm{m}^{2} \\ \text { initial water saturation } & 0.0 \\ \mathrm{~J} \text { - function = } & 0.01 / \mathrm{S}^{2} .\end{array}$

The permeability and the J-function were chosen in such a way to make the influence of the porous medium drastically apparent.

The numerical calculations were programmed in FORTRAN. The number of iterations needed to reach equilibrium was only slightly higher in the 'porous' case due to the updating of the pressure of the liquid phase at each iteration step.

\section{DISCUSSION OF RESULTS:}

The results of the numerical calculations for the two-component model system and the model porous medium are presented in Figs.4-9.

The linear theory can - as laid out before - be applied if the parameter $\varepsilon$ is small. $\varepsilon$ is, according to equ.(12), inversely proportional to pressure. The pressure dependence of $s$ worsens the situation. Fig. 4 gives a picture of this fact. For covering the entire influence of the porous medium on the condensation process one has to look at the order of magnitude of the capillary pressure or J-function itself (Fig.5). Close to the dew point the capillary pressure grows without limits. 


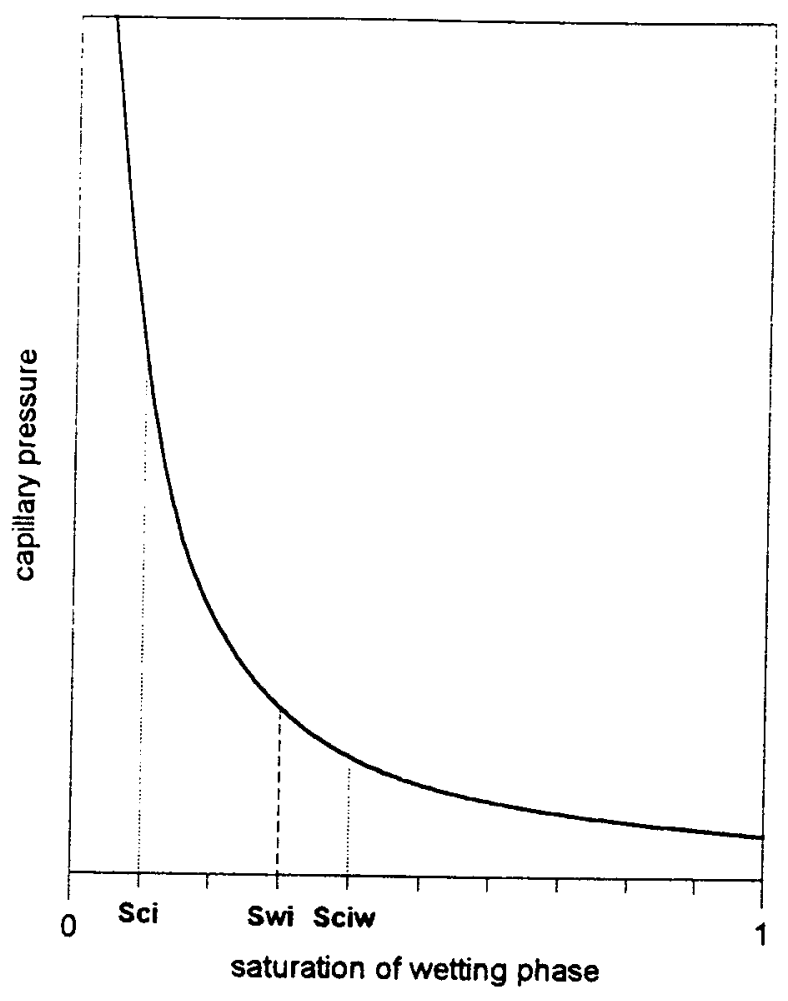

Fig. 1: Capillary pressure for different cases

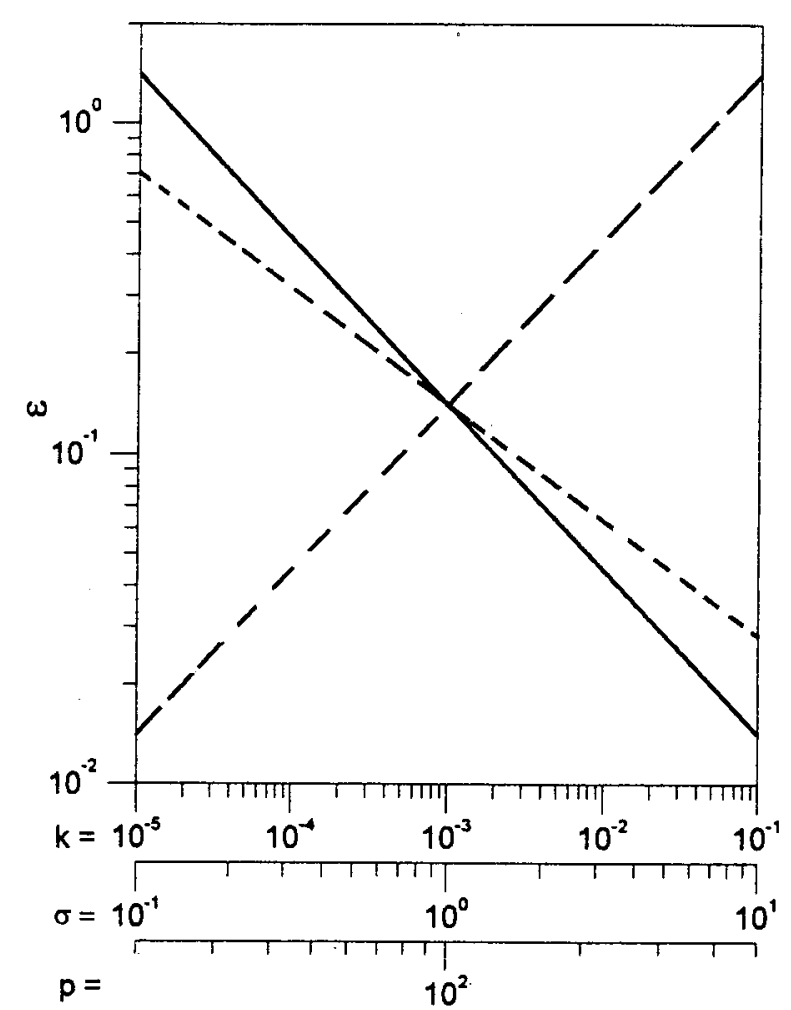

Fig.2: Dependence of $\varepsilon$ on permeability ( $\longrightarrow$ ). IFT $(---)$ and pressure $(\cdots . .$.$) .$

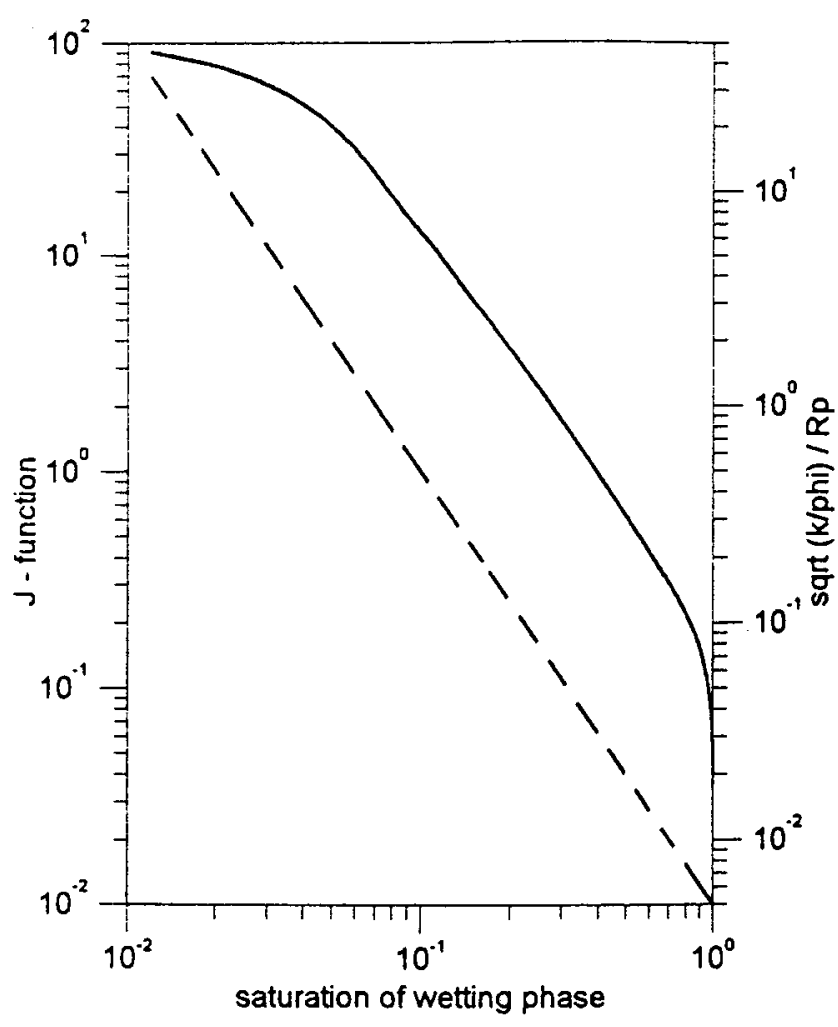

Fig.3: Characterization of the porous medium, $(--)^{-}$model J-function, ( - ) field Hoflein

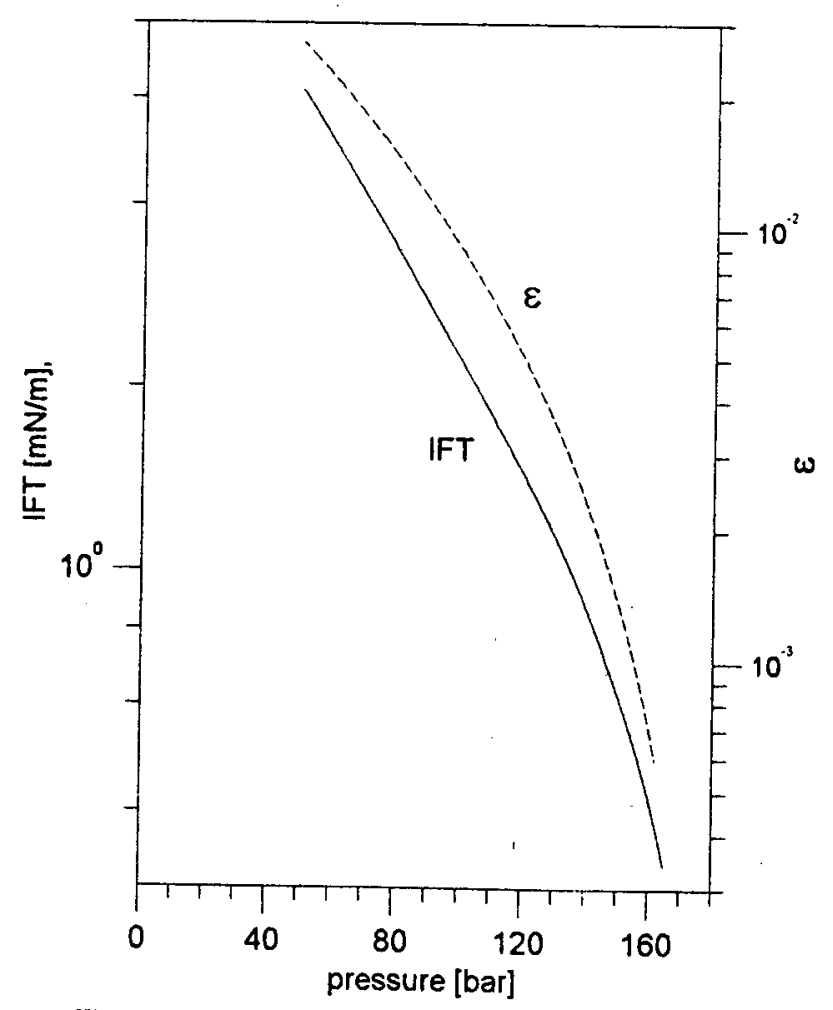

Fig.4: Interfacial tension for the system $\mathrm{C} 1-\mathrm{nC} 5$ and parameter $\varepsilon$. 


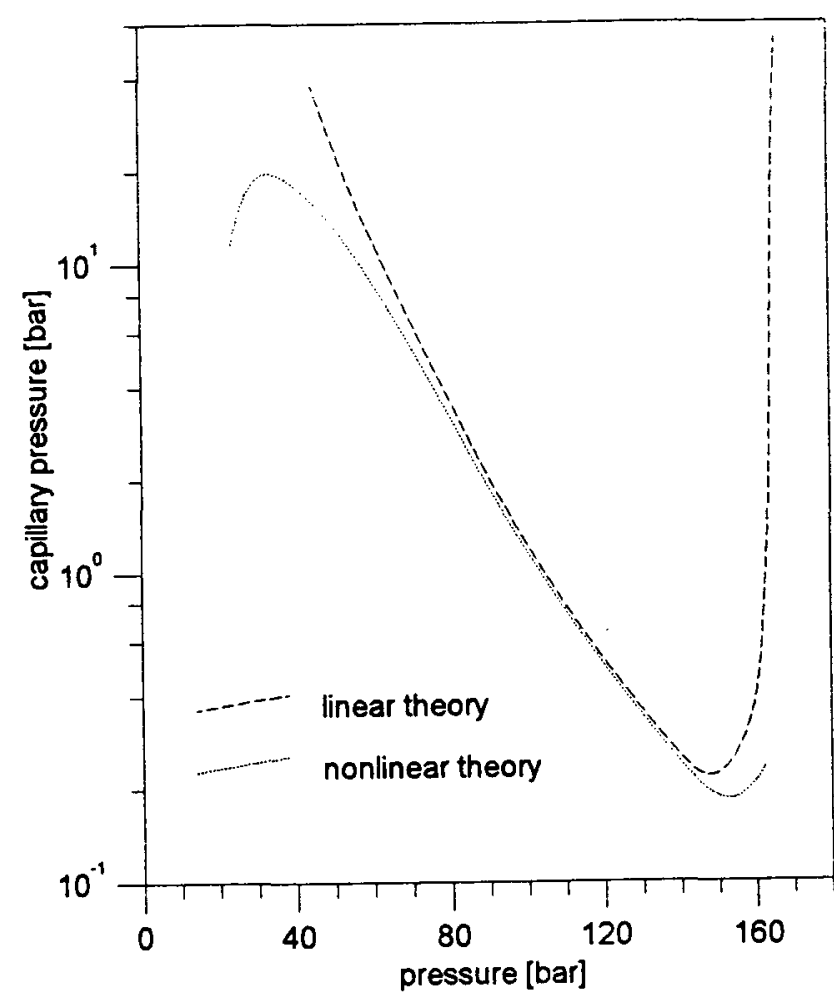

Fig.5: Capillary pressure due to the porous medium.

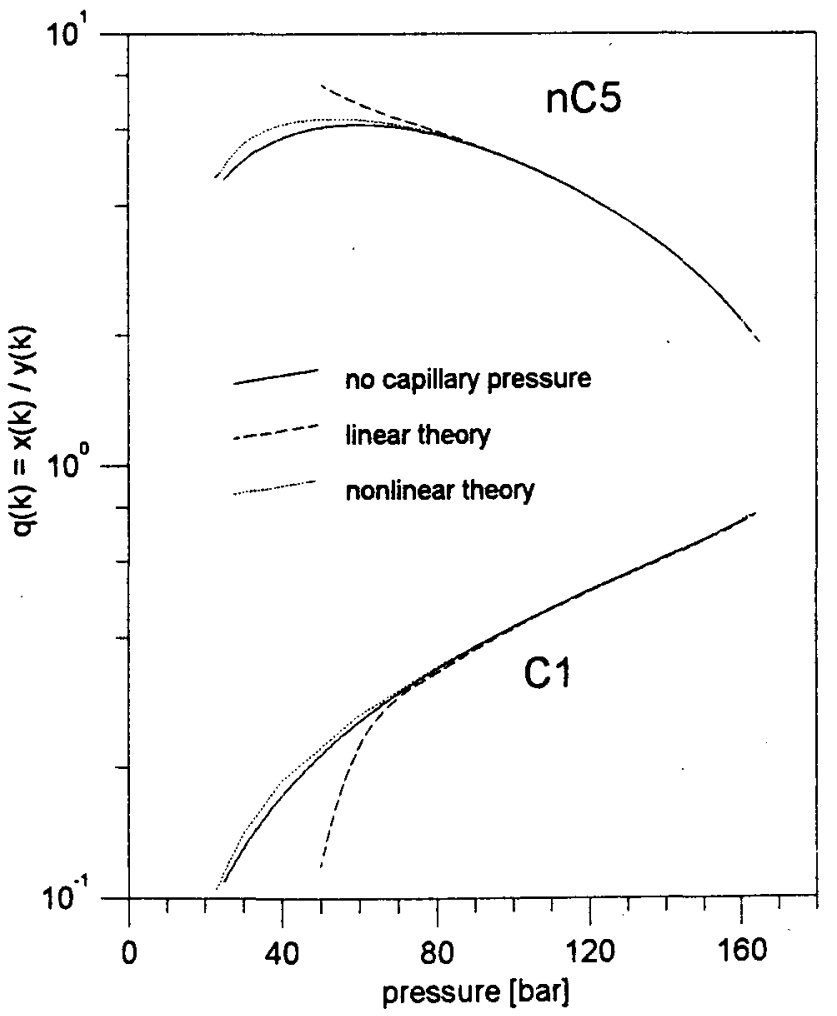

Fig.7: Equilibrium ratios for the system $\mathrm{C} 1-\mathrm{nC} 5$

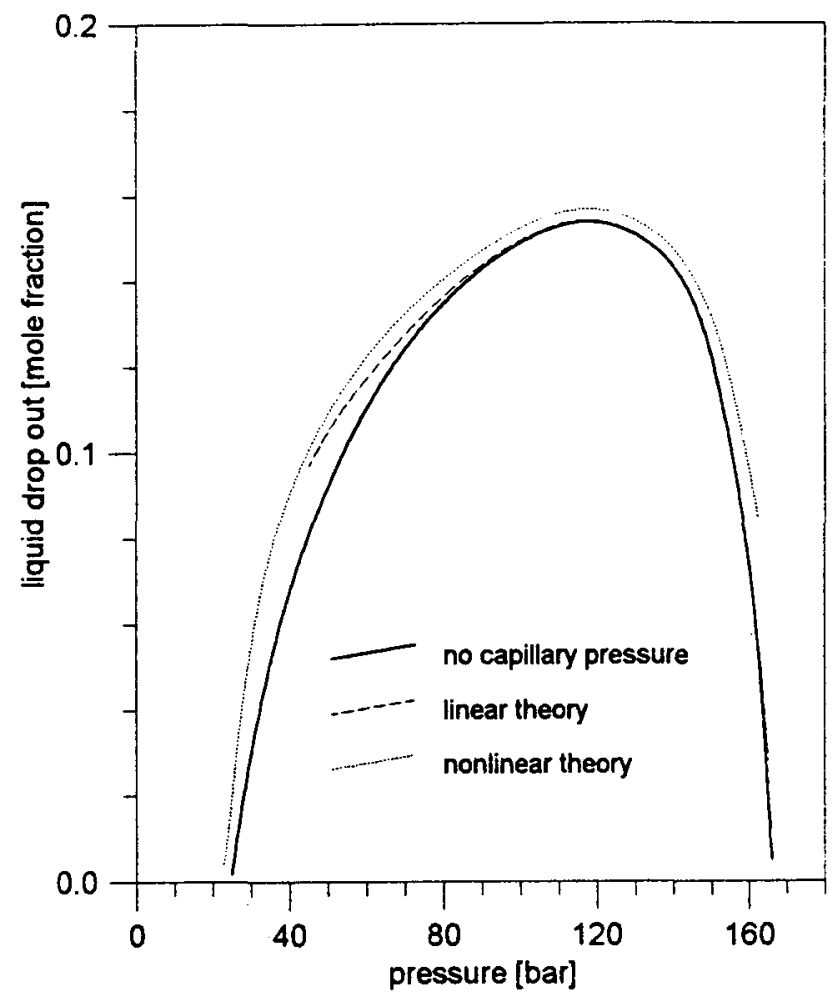

Fig.6: Liquid drop out for the system C1-nC5 (0.8:0.2) at $\mathrm{T}=80 \mathrm{C}$.

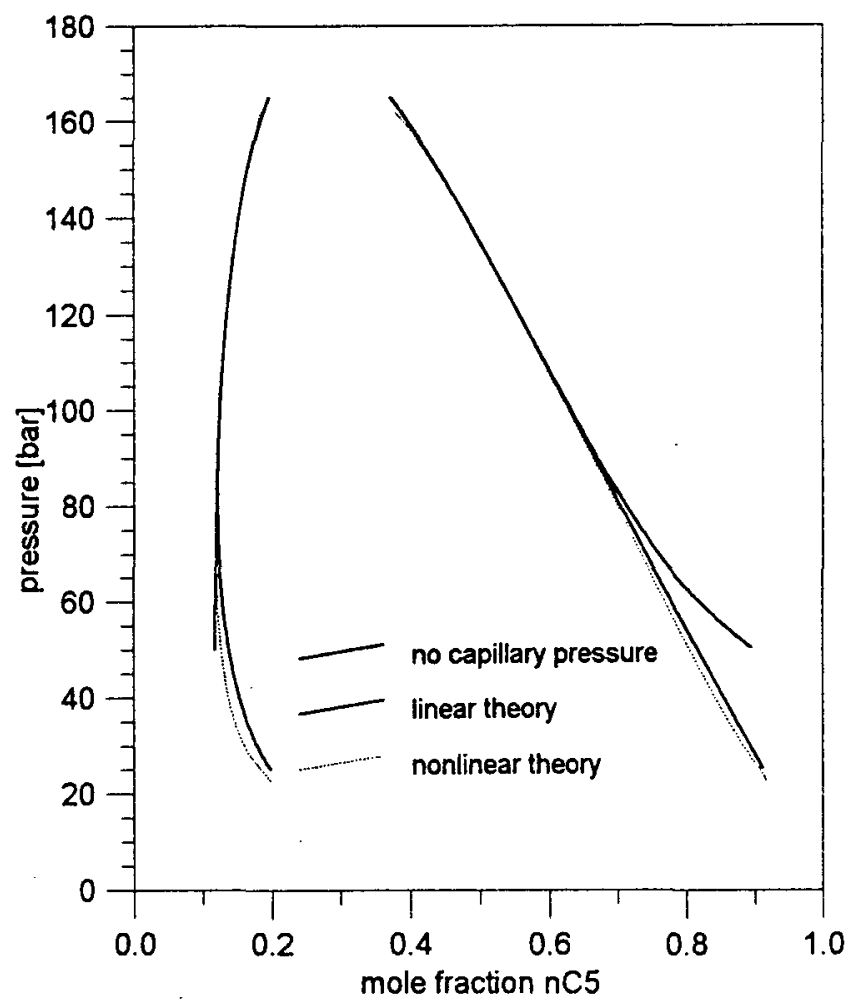

Fig.8: $p-x$ diagram for the system $\mathrm{C} 1-\mathrm{nC} 5$ at $\mathrm{T}=80 \mathrm{C}$. 


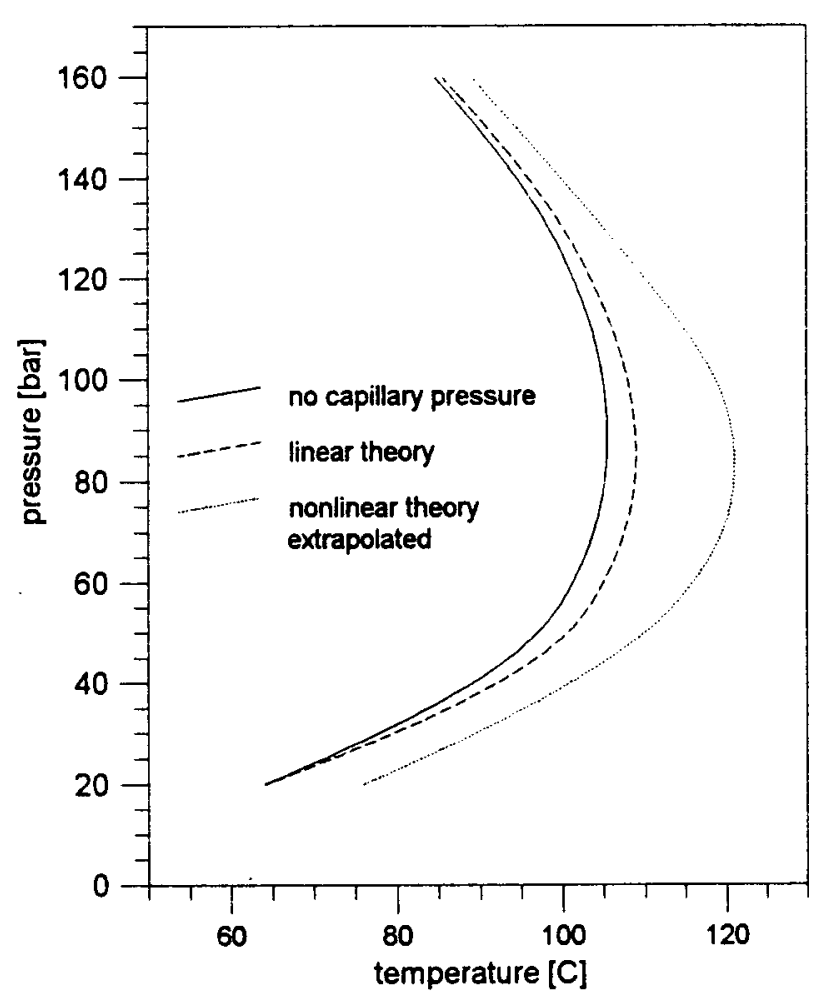

Fig.9: Phase envelope for the system C1-nC5 at $T=80 \mathrm{C}$.,

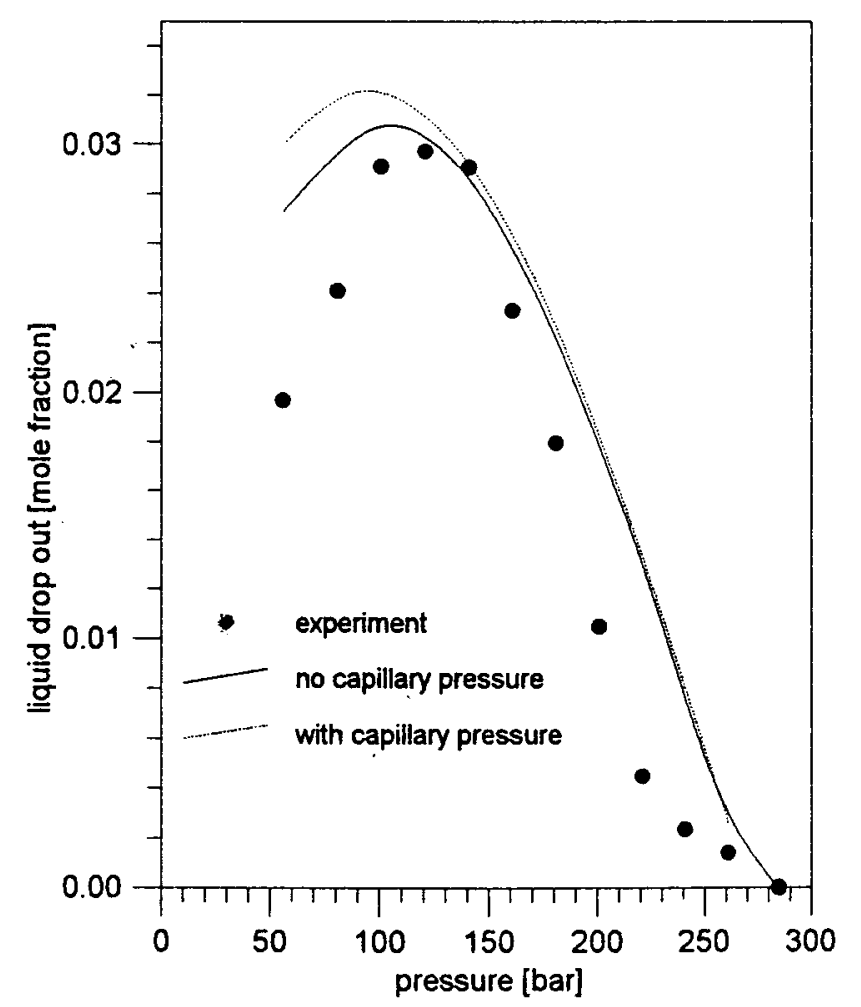

Fig.11: Liquid drop out for the system Höflein at $\mathrm{T}=78 \mathrm{C}$.

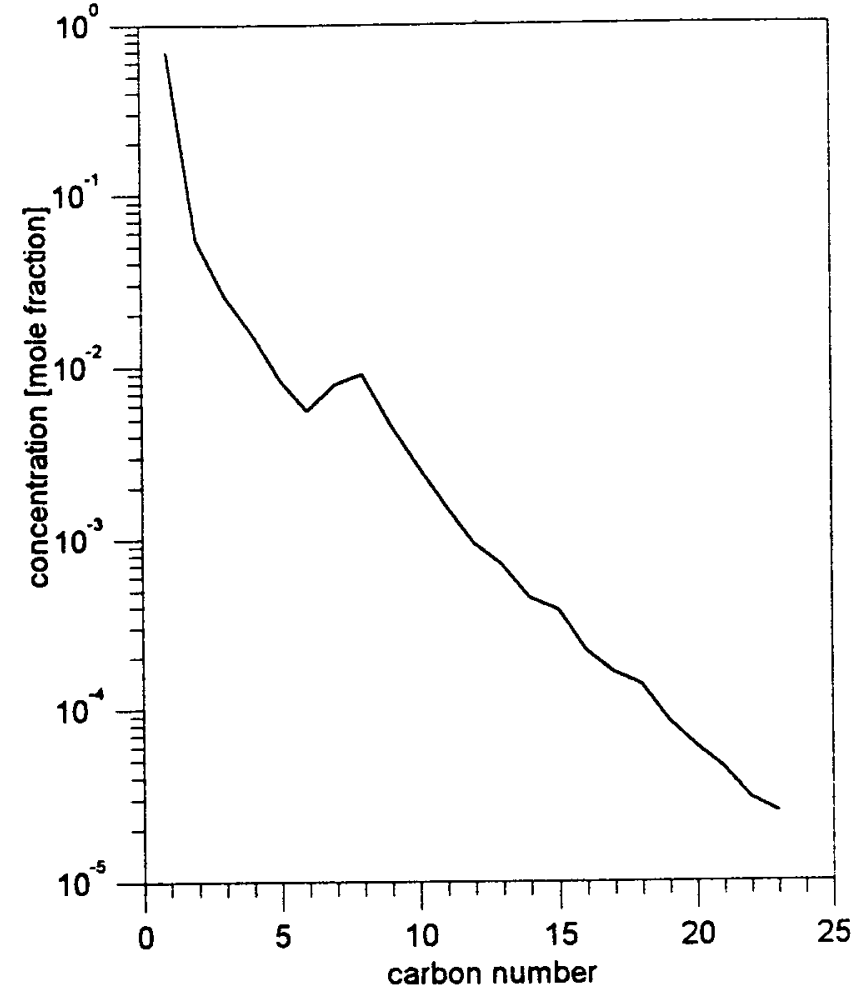

Fig.10: Hydrocarbon distribution in the field Höflein 